\title{
1 Dynamical Systems and Production Systems
}

\author{
D. Armbruster
}

Recent applications of nonlinear dynamics to three models of production systems are reviewed: (i) a re-organization procedure for bucket brigade systems is introduced. With such a procedure the bucket brigade self-organizes when a new worker is added whose skill level increases with time due to learning. (ii) Self-organization in chaotic networks of switched arrival systems is presented. (iii) A new continuum model of production flow through re-entrant factories leading to non-local nonlinear partial differential equations is discussed. Such a model may lead to fast simulations of supply chains as well as improved understanding of the dynamics of supply chains.

\subsection{Introduction}

This paper aims to give a review of some recent models for the organization and management of production systems, which were using ideas from dynamical systems theory. The topics that we will discuss are (i) the self-organization of workers along a linear production line using the organizing principles of a bucket brigade, (ii) chaotic dynamics in fluid models of production networks and (iii) nonlinear dynamics in a "traffic model" of a re-entrant factory. All these models have in common that the nonlinearity that makes them complex and interesting does not come from any nonlinear physical law. The nonlinearity typically is a result of policies or decisions that change the rules of the game. As a consequence, very often the underlying dynamical system is piecewise linear or even piecewise constant leading to hybrid dynamical systems. Unfortunately beyond some initial attempts [21,37] there is no comprehensive theory of hybrid dynamical systems available at the moment, which leads in general to ad hoc methods of analysis. On the other hand, the existing models indicate the importance of further research into a general theory of hybrid systems and even further into a general theory of the dynamics of decision systems. Rather than reporting in detail on previous work, I will in general simply state the major results and focus on extensions and open questions.

\subsection{The Bucket Brigade Production System}

Operational control in a linear production line is extremely important to optimize production. Typical studies focus on a fixed and optimal worker allocation that balances throughput along the line. As an alternative there is a growing interest to study operational control in systems with multi-skilled, flexible workers that are allowed to search for their own optimal place along

Nonlinear Dynamics of Production Systems. Edited by G. Radons and R. Neugebauer Copyright (C) 2004 Wiley-VCH Verlag GmbH \& Co. KGaA, Weinheim ISBN 3-527-40430-9 
a production line. In that case, instead of fixed work allocation, control is generated through a set of policies that tells each worker what to do "next".

The Toyota Sewn Products Management System (TSS) is one of the most widely studied architectures of this kind. TSS is employed regularly by manufacturers of sewn products in modules that are used in the finishing and assembly of cut parts into a subassembly or finished garment. The production modules are typically $\mathrm{U}$-shaped, and workers process garments as a team. In a TSS line, each worker picks up a task and processes (also carries) it at each station until he gets bumped by a downstream worker. There are no additional work in progress (WIP) buffers kept. The ordering of the workers has to be preserved but, other than that, workers are not restricted to any particular zones. When a worker comes to a busy station he must wait until the station becomes available; he also may not seek other work. The number of machines in a TSS line typically ranges from 2 to 16 with an average of 2.5 machines per worker [7]. In addition to the apparel and garment industry, TSS lines have also been shown to perform robustly in certain types of warehousing environments [5].

The term "bucket brigade" was coined by Bartholdi and Eisenstein [5] for TSS lines in which the workers are sequenced from slowest to fastest. The authors provided the first comprehensive analysis of the dynamics of such systems and showed that the bucket brigade is self-balancing; that is, eventually a stable partition of work among workers will emerge such that each worker repeatedly executes the same interval of work content. The basic assumptions in [5] are:

- work is continuous and workers can walk back and take over the work of their predecessors at any point;

- the work is deterministic and each worker has a velocity function that gives his/her instantaneous velocity at each point along the production line;

- processing times are deterministic; however, there is still variation due to the fact that workers have different skill profiles and, hence, different velocities at different tasks;

- workers walk back with infinite velocity (i.e. instantaneous reset of the line).

Such a setup will lead to a nonlinear dynamical system in the following way: consider $N$ workers along a production line. We model the line by the interval $0 \leq \xi \leq 1$ with $\xi=0$ denoting the start of production and $\xi=1$ indicating the end of the line. Let $x^{m}(t)$ be the position of the worker $m$ at time $t$ along the production line and $c_{m}$ her velocity. For the time being we assume that all velocities are constant along the whole production line. This can easily be changed.

From the time a new part is started on the production line by the first worker until the time that the last worker finishes her part, the time evolution of the positions of all $N$ workers is given by

$$
\begin{aligned}
& x^{1}(t)=c_{1} t, \\
& x^{2}(t)=c_{2} t+x_{0}^{2}, \\
& \cdots \\
& x^{N}(t)=c_{N} t+x_{0}^{N} .
\end{aligned}
$$


The time $\bar{t}$ to finish the next product is given by the equation

$$
x^{N}(\bar{t})=1 \text {. }
$$

Hence

$$
\bar{t}=\frac{1-x_{0}^{N}}{c_{N}} .
$$

The positions of all workers at the time $\bar{t}$ will be the starting point for the reset production line when all workers instantaneously walk back to pick up the part from their predecessor. Registering these positions leads to an obvious Poincaré map that relates the reset positions for finishing product $n$ to the reset position for finishing the next product $n+1$ :

$$
\begin{aligned}
& \mathbf{P}: \Sigma=\left\{\left(x^{1}(\bar{t}), x^{2}(\bar{t}), \ldots, x^{N-1}(\bar{t}), 1\right)\right\} \rightarrow \Sigma \\
& x_{n+1}^{1}=\frac{c_{1}}{c_{N}}\left(1-x_{n}^{N-1}\right), \\
& x_{n+1}^{2}=\frac{c_{2}}{c_{N}}\left(1-x_{n}^{N-1}\right)+x_{n}^{1}, \\
& \ldots \\
& x_{n+1}^{N-1}=\frac{c_{N-1}}{c_{N}}\left(1-x_{n}^{N-1}\right)+x_{n}^{1} .
\end{aligned}
$$

Bartholdi and Eisenstein [5] rigorously proved that the Poincaré map $\mathbf{P}$ has a globally stable fixed point if $c_{1}<c_{2} \cdots<c_{N}$. They also proved that the resulting throughput is optimal. Hence with a bucket brigade production rule, the production line organizes itself in an optimal way, obviating the need for intervention by management or for fixed work allocation rules. Recently we have extended this result in several directions [3, 4] which we discuss next. See also the related contribution by Bunimovich in this book [8].

\subsubsection{Re-ordering}

In the original bucket brigade setup, there is still management intervention in the form of determining the ordering of the workers along the production line. This is clearly a major problem since management will not always know the true ranking of worker speeds. In addition, worker speed will change not only stochastically, but also systematically. Workers will become faster (due for instance to learning) or slower (due for instance to health-related issues). In [4] we discuss the following re-order policy for bucket brigades: Whenever a worker gets blocked, he switches position with the blocking worker and continues to move ahead. At reset, every worker walks back until he encounters another worker along the production line. $\mathrm{He}$ will then take over the job from his predecessor, leaving the first worker to start a new product. The following lemmas will prove that, for a uniform bucket brigade (i.e. where the workers have constant speeds along the whole production line), this re-ordering algorithm will lead to a stable organization, corresponding to an ordering from slowest to fastest.

Lemma 1: A uniform bucket brigade that is not ordered slowest to fastest has an unstable fixed point. 
Proof: Assume a balanced bucket brigade, i.e. every worker repeatedly executes the same interval along the production line. Consider two neighboring workers. They comprise a twoworker bucket brigade. Assume worker A has a speed of $v_{\mathrm{A}}$ and worker B has a speed of $v_{\mathrm{B}}$. Call $p$ the handover point from worker A to worker B. Then

$$
p^{n+1}=\frac{v_{\mathrm{A}}}{v_{\mathrm{B}}}\left(c-p^{n}\right)
$$

if worker A comes before worker B along the production line. The constant $c$ describes the handover point for worker $\mathrm{B}$ and is unimportant. If $v_{\mathrm{A}}<v_{\mathrm{B}}$ the fixed point of the linear map (1.2) is stable whereas for $v_{\mathrm{A}}>v_{\mathrm{B}}$ the fixed point is unstable.

Lemma 2: A balanced uniform bucket brigade that has an unstable fixed point shows blocking. Proof: The nature of blocking is that the worker that gets blocked slows down to the speed of the blocking worker. The system (1.1) with blocking can be written as

$$
\mathbf{x}^{n+1}=\mathbf{A}^{i} \mathbf{x}^{n}+\mathbf{c}^{i}
$$

where $\mathbf{x}$ is the vector of all reset positions, $\mathbf{A}^{i}$ a constant non-singular matrix and $\mathbf{c}^{i}$ a constant vector. The index $i$ counts the different instances of possible blocking and represents different initial domains that have more or less instances of blocking. As a result the system (1.3) is a piecewise-linear system. The definition of a fixed point implies that there is no blocking. We choose $i=1$ to be that case. By assumption that fixed point is unstable. Since the dynamics restricted to the domain of the $i=1$ system is linear, any starting point near that fixed point will eventually reach the boundary of that system. Blocking occurs at that point.

Lemma 3: A generic bucket brigade with the above reset rule will converge to the stable fixed point.

Proof: Since the system (1.1) is piecewise linear, there are generically no other attractors than fixed points completely inside just a single linear domain. Blocking can only happen if a trailing worker $n$ has a speed $c_{n}$ that is larger than the speed of the following worker $c_{n+1}$. In that case the worker order will be reversed. Hence the re-order rule can never switch a worker with lower speed into a higher position. This is a terminating process that leads to the stable ordering from slowest to fastest.

As a result of these lemmas, there is no management intervention necessary to balance the bucket brigade. As long as the order of the workers' speeds is uniform along the production line, the re-ordering rule will seek out the balanced line. Non-uniform bucket brigades (i.e. workers' speed order not constant along the production line) have the possibilities of multiple fixed points. Hence the above arguments will not work in general.

\subsubsection{Non-constant Speeds}

The assumption of a fixed worker ordering along a production line is too restrictive in many settings. This is true especially for production lines that have different types of tasks or for warehouse picking, where a worker will be familiar with one part of the inventory but very unfamiliar with another part of the warehouse. In our paper [3], we study two workers who have varying levels of specialization at different tasks. As as result no worker speed uniformly dominates the other over the whole production line. To illustrate the type of dynamics that happens in this case, we discuss a case study for the passing case. 
Let's suppose we have two workers, A and B. For convenience, we refer to worker A as a male and worker B as a female. We scale work content and time such that worker B has a uniform speed along the production line which we set to 1 . We assume that the speed of worker A is a function of his location on the line. To ensure that worker B's speed does not uniformly dominate that of worker $\mathrm{A}$, we assume that worker $\mathrm{A}$ has speed $c_{1}$ on the interval $[0, X)$ and $c_{2}$ on the interval $[X, 1]$ with $c_{1}<1<c_{2}$. A worker may overtake the next worker temporarily. However, a fixed worker order is assumed and we call the system unbalanced if that worker order is not true at the time of reset of the production line. Consider the case when worker $\mathrm{A}$ is placed at the end of the line. We define $x_{\mathrm{B}}(t)\left(x_{\mathrm{A}}(t)\right)$ as the position of worker $\mathrm{B}$ (worker A) along the production line at time $t$. Since the speed of worker B is equal to 1 for all portions of the work we find

$$
x_{\mathrm{B}}(t)=t \text { for all } t \geq 0 \text {. }
$$

For worker A, $x_{\mathrm{A}}(t)$ will change as a function of where he started. Let $x^{0}$ denote the location that worker A took over the first job, that is, $x^{0}:=x_{\mathrm{A}}(0)$. If worker A starts at a point after the break point $X$ (i.e. $x^{0}>X$ ), then the location of worker $\mathrm{A}$ at time $t$ will be governed by:

$$
x_{\mathrm{A}}(t)=x^{0}+c_{2} t \text { for all } t \geq 0 .
$$

If worker A takes over the job at some point before $X$ (i.e. $x^{0} \leq X$ ) then we get:

$$
x_{\mathrm{A}}(t)= \begin{cases}x^{0}+c_{1} t & \text { for } t<t_{X}, \\ X+c_{2}\left(t-t_{X}\right) & \text { for } t \geq t_{X},\end{cases}
$$

where $t_{X}$ is the time it takes worker A to get to $x_{\mathrm{A}}=X$ :

$$
t_{X}=\frac{X-x^{0}}{c_{1}} .
$$

It takes worker $\mathrm{A} \bar{t}_{1}$ time units to complete the first job, which is found by setting $x_{\mathrm{A}}(t)=1$ :

$$
\bar{t}_{1}= \begin{cases}\frac{1-x^{0}}{c_{2}} & \text { for } x^{0}>X \\ t_{X}+\frac{1-X}{c_{2}}=X\left(\frac{1}{c_{1}}-\frac{1}{c_{2}}\right)-\frac{x^{0}}{c_{1}}+\frac{1}{c_{2}} & \text { for } x^{0} \leq X\end{cases}
$$

Again we get a piecewise-linear map

$$
x^{n+1}:=x_{\mathrm{B}}\left(\bar{t}_{n}\right)= \begin{cases}\frac{1-x^{n}}{c_{2}} & \text { for } x^{n}>X, \\ X\left(\frac{1}{c_{1}}-\frac{1}{c_{2}}\right)-\frac{x^{n}}{c_{1}}+\frac{1}{c_{2}} & \text { for } x^{n} \leq X .\end{cases}
$$

The map is piecewise linear with slopes $-1 / c_{1}<-1$ and $-1 / c_{2}>-1$. The elbow of the map is at $x^{n}=X$ with $f(X)=(1-X) / c_{2}$. Clearly there always exists exactly one fixed 


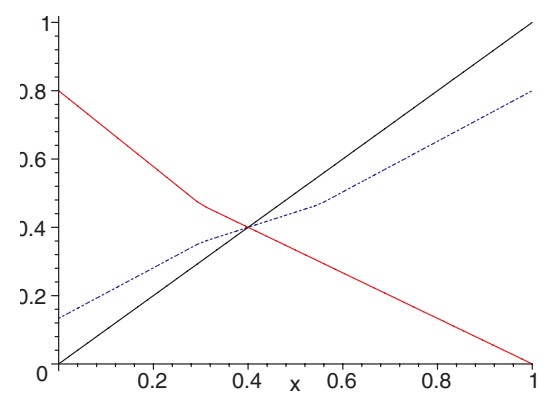

(a) $X \leq x_{\mathrm{s}}, M \leq 1$

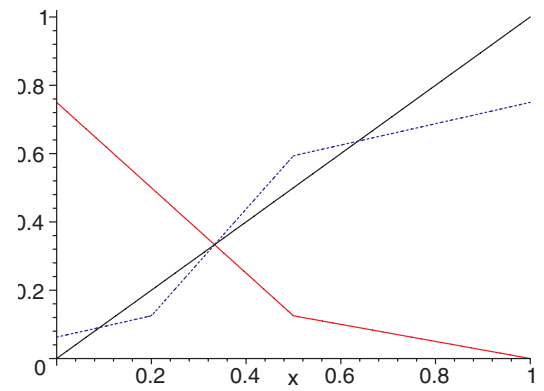

(c) $X>x_{\mathrm{s}}, M \leq 1$

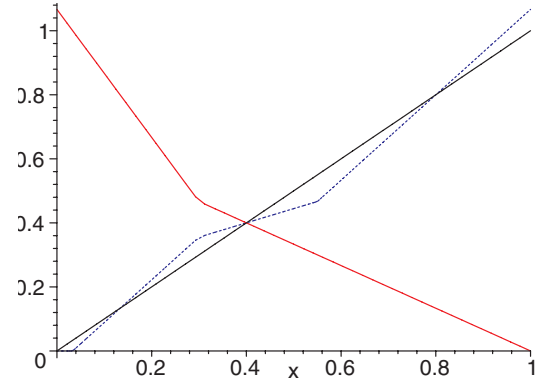

(b) $X \leq x_{\mathrm{s}}, M>1$

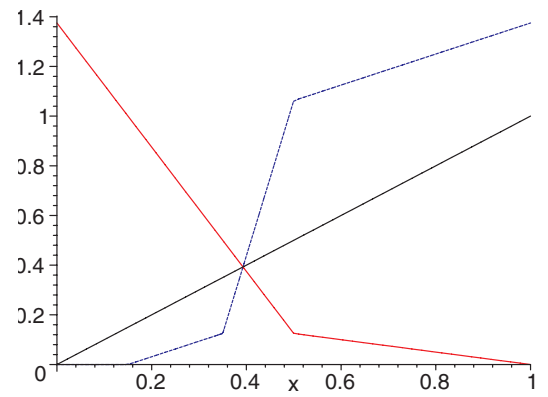

(d) $X>x_{\mathrm{s}}, M>1$

Figure 1.1: All possible dynamics for the passing case when worker $A$ is placed at the end.

point of that map that we call $x_{\mathrm{s}}$. If $X \leq x_{\mathrm{s}}$ (i.e. the slope at $x_{\mathrm{s}}$ is equal to $-1 / c_{2}>-1$ ), then the fixed $x_{\mathrm{s}}$ point is stable. If $X>x_{\mathrm{s}}$, the fixed point $x_{\mathrm{s}}$ is unstable.

Figure 1.1 shows all four possible cases for the dynamics of the bucket brigade. Each of the subfigures shows: (i) the Poincaré map $x^{n+1}=f\left(x^{n}\right)$ (depicted by the dashed line), (ii) $x^{n+2}=f^{2}\left(x^{n}\right)=f\left(f\left(x^{n}\right)\right)$ and (iii) the $y=x$ line as the diagonal. Figure 1.1a and 1.1b show the case of $X \leq x_{\mathrm{s}}$, which results in a stable fixed point. Figure 1.1c and 1.1d show the case of $X>x_{\mathrm{s}}$, which results in an unstable fixed point. The critical quantity for the dynamics in both cases is $M$, which is defined as the time that worker A would need to get through the whole production line. Then, $M=f(0)$ can be found as

$$
M=\frac{X}{c_{1}}+\frac{1-X}{c_{2}} .
$$

Note that if $M>1$, then worker B is faster on average than worker A.

- $X \leq x_{\mathrm{s}}$, stable fixed point:

- Figure 1.1a shows the case with worker A faster on average than worker $\mathrm{B}(M \leq 1)$. We see that the stable fixed point attracts all initial conditions. 
- Figure 1.1c shows the case with worker B faster on average than worker A $(M>1)$. In this case, the fixed point is still stable, but there also exists a period-two orbit that is unstable. That is, all initial conditions inside the period-two orbit converge to the fixed point and all initial conditions outside the period-two orbit lead to a situation where worker B finishes her job before worker A does, which means that A is not fast enough at the end of the production line to maintain the ordering.

- $X>x_{\mathrm{s}}$, unstable fixed point:

- Figure 1.1b shows the case with worker A faster than worker $\mathrm{B}$ on average $(M \leq 1)$. We see that the fixed point is unstable; however, there exists a stable period-two orbit that attracts all initial conditions and preserves the order of the bucket brigade.

- Figure 1.1d shows the case $X>x_{\mathrm{s}}$ with worker B faster than worker A on average $(M>1)$. In this case, the fixed point is unstable and all initial conditions eventually lead to a situation where worker B finishes her job before worker A does and, hence, the order of the bucket brigade is not preserved.

This case study can be generalized to the following theorem [3].

\section{Theorem}

The following results hold true for the dynamics of two-worker bucket brigades with passing, regardless of worker ordering:

(i) If the worker at the end is on average the faster worker then either the fixed point is globally stable and attracts all initial conditions or the fixed point is unstable, in which case there exists a stable period-two orbit that attracts all initial conditions.

(ii) If the worker at the end is on average the slower worker then either the fixed point is unstable and all initial conditions lead to an unbalanced bucket brigade or the fixed point is stable and there exists an unstable period-two orbit. All initial conditions inside the period-two orbit lead to a balanced line at the fixed point; initial conditions outside the period-two orbit lead to a reversal of the finishing order of the bucket brigade.

\subsubsection{Bucket Brigades and Learning}

Bucket brigades are typically set up for relatively low-skilled work. Low pay and non-existing benefits often lead to a large worker turnover [16]. Hence very often an existing bucket brigade has to incorporate a new member who is unskilled and at the time a slow worker. As he gains experience, his skills improve and so does his production speed. Recently we have studied the implication of learning on the stability of a bucket brigade [4]. The most important feature of the models of bucket brigades with learning is the fact that the dynamical systems become non-autonomous. To be specific, we consider a well-ordered, uniform bucket brigade of $N$ workers arranged from slowest to fastest: $c_{1}<c_{2}<\cdots<c_{N}$. One new worker will be added at an arbitrary place in the worker ordering. We adopt the re-order policy discussed previously. Hence workers can pass each other and handovers will always be done to the nearest worker upstream. We assume a starting velocity for the new worker: $v_{1}<c_{i} \forall i$, constant and a 
potential maximal velocity $v_{\mathrm{h}}$. The worker learns only on those parts of the production line where he/she has worked.

Hutchinson, Villalobos and Bernvides suggested the following model for the increase in worker speeds due to learning [16]:

$$
\begin{aligned}
v_{n} & =v_{\mathrm{l}}+\left(v_{\mathrm{h}}-v_{\mathrm{l}}\right)\left(1-e^{-t_{n} / \tau}\right), \\
t_{n} & =\sum_{i=0}^{n} t_{i} .
\end{aligned}
$$

\section{Theorem}

The bucket brigade will self-organize to the same throughput, independent of initial conditions (initial worker ordering).

Figure 1.2 shows the asymptotic velocities as a function of the positions along the production line of a four-worker bucket brigade with one new worker added. The constant speeds of the existing bucket brigade are

$$
\left(c_{1}, c_{2}, c_{3}, c_{4}\right)=(1.3,1.6,2.0,2.3)
$$

the starting speed for the new worker is $v_{1}=1$ and the limiting speed for the new worker is $v_{\mathrm{h}}=1.8$. We see that the new worker typically only learns significantly on a finite interval. That interval represents the part of the production line that he will cover as part of the work allocation for the stable fixed point of the bucket brigade. Note also that workers typically stay in the original order although occasionally they change order after a few iterations. Figure 1.2e shows such a case where the initial position of the new worker was last but he switched to second to last. This is reflected in some initial increase in velocity close to the end of the production line. It is instructive to place the new worker into a position where its limiting speed will lead to an instability of the bucket brigade. Assume the limiting speed of the new worker is significantly higher than the speeds of the workers around him. In that case, the new worker will cover larger and larger parts of the production line, shifting his region of highvelocity motion further and further out. Eventually the resulting bucket brigade will become unstable, leading to first periodic behavior and then to a re-ordering due to the fact that the old order cannot be sustained at reset. Figure 1.3 shows the transition points between the various workers as a function of time. We see that an initial order slowly becomes unstable to a beautiful period-doubling cascade until the system resets on a new order that dynamically creates a fixed point. What looks like a period-doubling cascade is really a transient that evolves slowly through the quasi-steady periodic orbits.

\subsection{Fluid Models of Production Networks}

There are currently two major approaches to simulate production flows: discrete event simulations (DES) and fluid networks. DES have successfully been used in large simulations for semiconductor factories (for instance $[10,12]$ ) but typically are very time consuming. An alternative is the use of fluid models as discussed in [11, 19], etc. Fluid models come from traffic theory and were introduced by Newell $[28,30]$ to approximately solve queueing problems. 


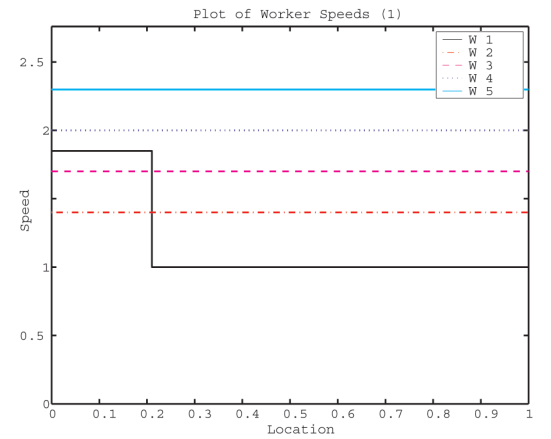

(a) New worker at position 1

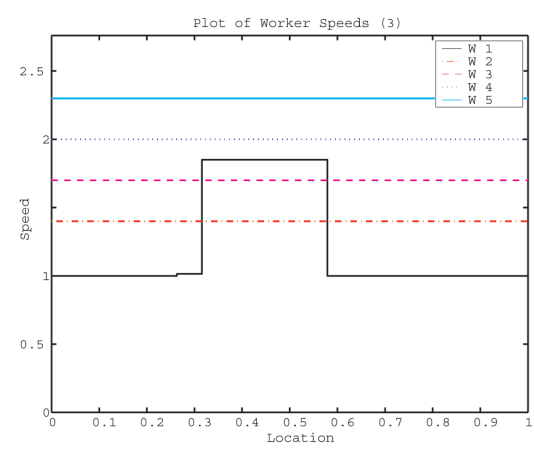

(c) New worker at position 3

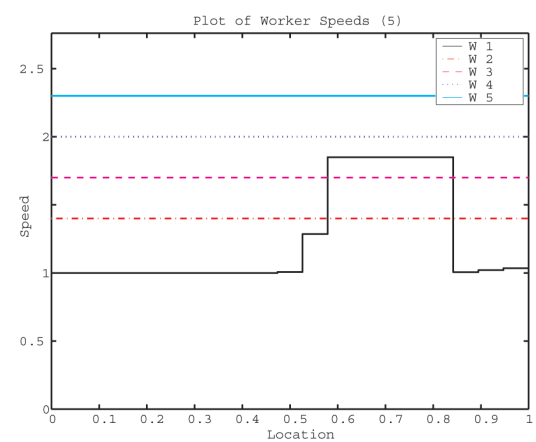

(e) New worker at position 5

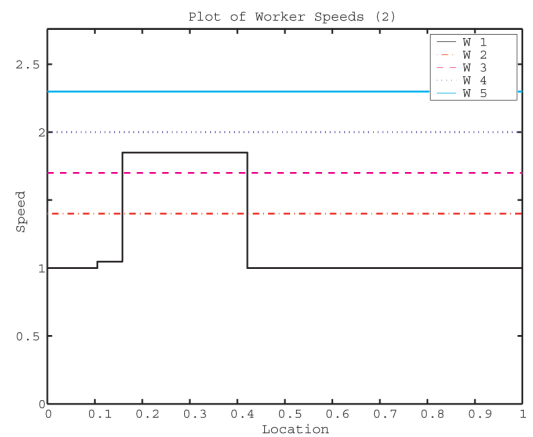

(b) New worker at position 2

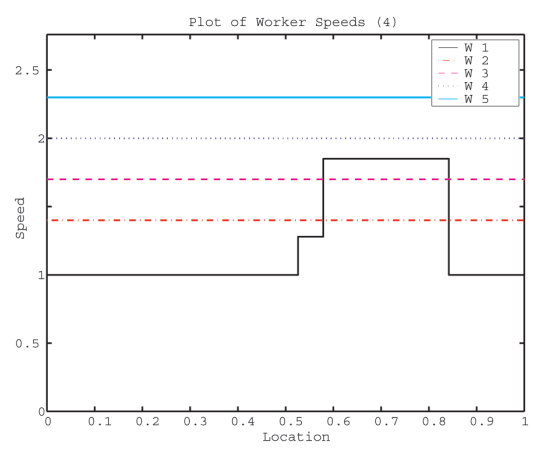

(d) New worker at position 4

Figure 1.2: Asymptotic velocity distributions for adding a new worker at different places in an existing bucket brigade. 


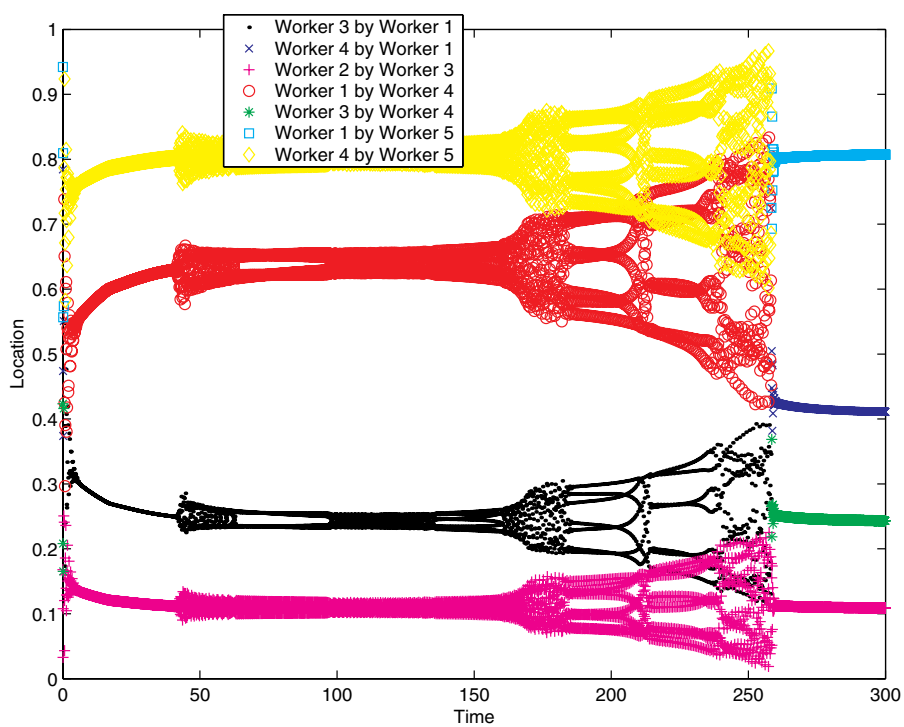

Figure 1.3: Handover points for a four-worker bucket brigade with one additional learner (worker 1).

They consider the length of a queue $x(t)$ as a continuous variable whose rate of change is given by:

$$
\frac{d x}{d t}= \begin{cases}\lambda(t)-\mu(t) & \text { for } x(t) \neq 0, \\ 0 & \text { for } x(t)=0,\end{cases}
$$

where $\lambda(t)$ is the arrival rate and $\mu(t)$ the processing rate of the queue. Notice that while in general $\lambda$ and $\mu$ are random variables and hence Eq. (1.5) represents a stochastic differential equation, the usual models assume nice distributions for the arrival and departure processes and hence use average start and processing rates. This basic building block for a queue can be connected to a work-conserving fluid model by feeding the outflux of each queue into other queues. The basic nonlinearity of this model stems from the fact that there is not a simple righthand side to Eq. (1.5) but a discontinuous flow. In addition scheduling policies will influence the influx rate $\lambda$ and the production rate $\mu$. Dai and Weiss [11] have analyzed the relationship between the stability of the fluid model and the stability of scheduling policies for the associated queueing networks. Here stability of the fluid model is represented by the boundedness of the fluid variables for a given influx $\lambda(t)$ which is assumed to be less than the smallest processing rate $\mu_{i}(t)$ of any queue in the network. Stability in the queueing theory sense is given by a unique stationary distribution $\psi$ for the underlying stochastic process describing the queueing network. Dai and Weiss [11] in particular showed that a queueing discipline is stable if the corresponding fluid model is stable.

The most well-known fluid model that showed chaotic dynamics is called the switched arrival system [9]. A flow model for $N$ parallel machines and one switching server that distributes work over the machines is considered. Work drains at a constant work rate while 


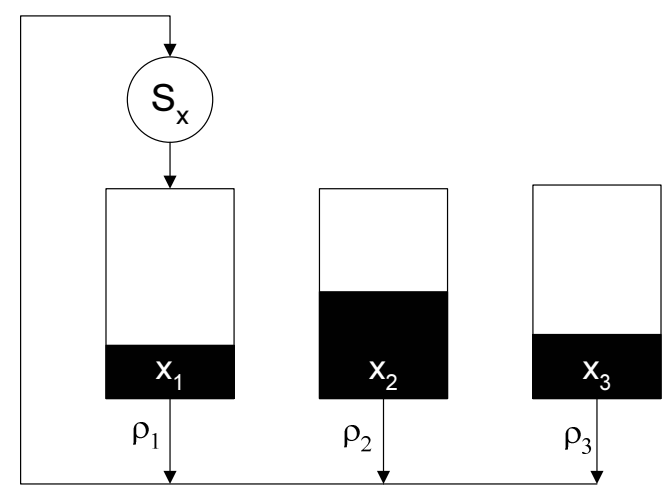

Figure 1.4: A switched arrival system for three machines.

the server continues to fill a buffer until another buffer empties (see Fig. 1.4). At that moment the server switches instantaneously to the empty buffer. The rate at which the server fills a buffer is equal to the sum of all the work coming out of all the buffers. This models a policy known in industrial engineering as the CONWIP policy (constant work in progress) which releases a new product into a machine or factory once a finished product has been released. Hence the system is closed and the total amount of work in the system is constant. Let $x_{i}(t)$ denote the amount of work in buffer $i$ at time $t$. We choose the total amount of work in all of the buffers to be 1 , i.e.

$$
\sum_{i=1}^{N} x_{i}=1 \text {. }
$$

We also choose the time to produce the total amount of work as the characteristic time and set it to 1 also. Hence the production rates $\rho_{i}$ satisfy

$$
\sum_{i=1}^{N} \rho_{i}=1 .
$$

Let $j$ be the position of the server. The server switches to the first buffer that empties. This happens after a time

$$
\tau=\min _{i \neq j}\left\{x_{i}(t) / \rho_{i}\right\}
$$

For $t_{0} \leq t \leq t_{0}+\tau$ the buffer state is determined by the following linear equations:

$$
x_{i}(t)= \begin{cases}x_{i}\left(t_{0}\right)-\rho_{i}\left(t-t_{0}\right) & \text { for } i \neq j, \\ x_{i}\left(t_{0}\right)+\left(1-\rho_{i}\right)\left(t-t_{0}\right) & \text { for } i=j .\end{cases}
$$

When this system is sampled at the times when a buffer empties the continuous model becomes a discrete-event model of the form

$$
G(x)=x+\min _{k \neq j}\left(\frac{x_{k}}{\rho_{k}}\right)\left(\mathbf{1}_{j}-\rho\right),
$$


where $\mathbf{1}_{j}$ is a vector with all zeros except for a 1 in the $j$ th position and $\rho$ is a vector containing the work rates $\rho_{i}$. The buffer state evolves on the simplex $\sum x_{i}=1$. For $N=3$ machines this simplex becomes an equilateral triangle and the hybrid system $G(x)$ maps the boundaries of the triangle onto each other. Obviously each individual map in $G(x)$ is expanding on the average with a rate of 2 . Hence, no matter what the production rates of the different machines are, there is always at least one region in phase space that is chaotic. Whether that region is a chaotic attractor or just leads to transient chaos depends on the details of the production rates. For instance, for the completely symmetric case when all the machines are identical and hence the production rate is $\rho=1 / 3$, the expansion is constant everywhere and the associated Lyapunov exponent is given as $\ln 2$. Peters et al. [33] have recently analyzed the bifurcations associated with varying the individual production rates of the machines. Katzorke et al. [18, 36] have studied a discretized version of this problem representing a discrete order flow. They show that the dynamics becomes periodic for rational production rates. Control schemes employing finite buffer sizes [15], setup times switching from one buffer to another [38, 39] or timed idling of one machine $[18,35]$ have been discussed.

One major drawback of the switched arrival system is the fact that the chaotic dynamics is strictly internal to the production - the total throughput through the set of machines is always constant and does not reflect the chaotic dynamics. Rem and Armbruster [35] introduced switching or maintenance time into the model. The basic idea is that the server cannot instantaneously switch from one machine to the other but needs a certain setup time during which the machine will not produce. In that way, the system will lose production whenever a queue is empty. The outflux values will typically not be chaotic but switch between a few discrete levels. However, the time between switches will become chaotic. Coupling switched arrival systems horizontally and vertically now leads to a much more realistic model of a layered production system. The analysis in [35] shows that the average throughput for the different layers will self-adjust to a steady state, although the dynamics of each individual layer is chaotic. Figure 1.5 shows the aggregate work levels in a three-layer, three-machine network working under the switched arrival protocol and with a CONWIP policy.

Coupling the switched arrival systems to a network opens up many new and exciting research avenues:

- The consequences of self-organized production networks are intriguing. So far, a typical production network is set up to have a constant flux through the network. This is typically done by expensive studies that determine the actual throughput at any particular station. Observed bottlenecks are identified and additional resources are allocated to them if possible. If production networks are able to organize themselves, that would reduce the need for difficult measurements and adjustments. It is interesting to note that the theme of "self-organization", which is the major rationale for the linear bucket brigade production system in the last section, is picked up in these production networks too. Further studies on the performance of self-organized networks and their stability towards systematic and stochastic parameter changes will be very important.

- The production networks are natural examples for a merging of the concepts of control of chaos [32] and chaotic synchronization [34]. So far, individual layers of machines have been controlled to optimal trajectories. On the other hand, while the network does not seem to synchronize completely, it nevertheless exchanges information between layers that 


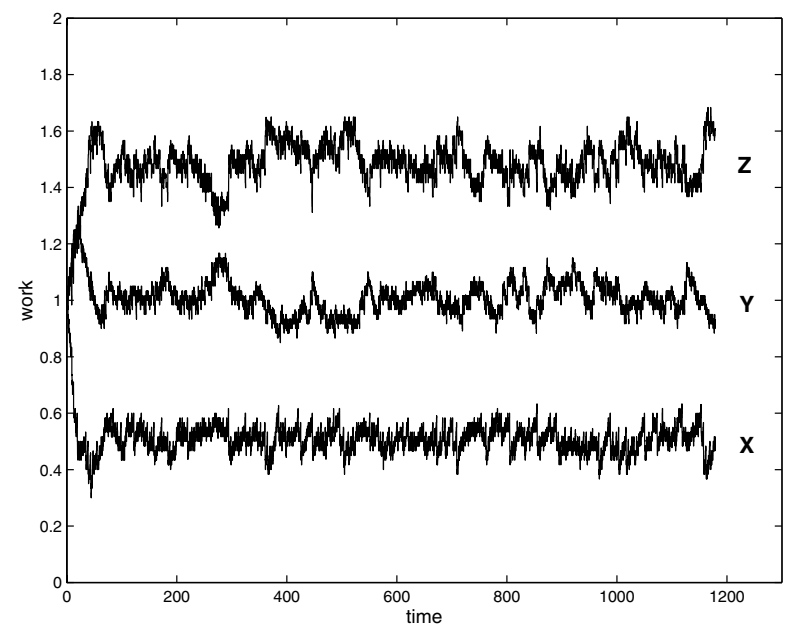

Figure 1.5: Work levels as a function of time for the three-layer system.

leads to the described self-organization. An obvious extension of the concepts of control and synchronization in chaotic systems is to determine whether there exist parameter regimes and production policies such that by controlling one layer in the network to an optimal trajectory, the other layers will become synchronized to that optimal dynamics. It seems obvious that by controlling one layer you will not be able to control a very large network but it is unclear whether there exists a neighborhood of layers that can be controlled. Issues of global vs. local control will be studied. Work along these lines is in progress.

\subsection{Dynamics of Supply Chains}

In recent years the focus of research into production dynamics has switched from modeling and optimizing individual factories to the study of a whole supply chain from the supplier's supplier to the customer's customer. One of the major open problem in that field is the question of how to scale up the simulation and analysis that worked well for an individual factory to the much larger problem of a whole supply chain. This fact was acknowledged by NSF when they started an RFP entitled "Scalable Enterprise Initiative" [31] that called for new ideas to simulate production flows in large systems. One typical response to that problem is to use discrete event simulators which have been quite successful as models for individual factories to model a whole supply network. The major problem with such an approach is that typical simulations for reasonably complicated production (e.g. in the semiconductor industry) take much too long. As a result, DES are not a useful tool for fast evaluations of management and policy decisions. Asynchronous and parallel simulations [22], together with the usual increases in raw computer power, may over time solve that problem. 
An alternative approach has been developed recently $[1,2]$ extending concepts from gas dynamics and traffic theory to production flows (see also [20, 14]). This approach not only will allow very fast simulations, it also allows for hierarchical models that, at least on their lowest levels, can be understood qualitatively, leading to insight into the dynamics of supply chains. While fluid systems (see Sect. 1.3) consider the work in the factory as a continuous variable, they still describe the flow through the factory by a discrete graph: work moves from one machine to another, moving from one node in a graph to the next. In order to generate a simple universal model for flows in a factory we assume that a product going through our factory undergoes many steps and that a limiting description of a continuous production line is a reasonable model. In that way we actually merge features of the underlying models of the bucket brigade and the fluid models. As a result, the relevant quantity moving through a factory is the work density (WIP density) $\rho(\xi, t)$ describing the amount of parts at time $t$ at completion stage $\xi$. Again, $\xi=0$ denotes the beginning of the factory while a part at $\xi=1$ is completed. A work-conserving production hence naturally leads to a conservation law for the WIP density:

$$
\rho_{t}+(v(\rho) \rho)_{\xi}=0,
$$

where $v(\rho)$ describes the velocity of the product moving in the factory. The exact nature of the transport velocity $v(\rho)$ is the major modeling issue. Following adiabatic gas theory or Lighthill and Whitham's [25] traffic model we may describe the functional dependence of the velocity via a state equation

$$
v=f(\rho) \text {. }
$$

We note that the units of $\rho$ are [parts][stage] and the units of $v(\rho) \rho$ are [parts][time]. This suggests that in conventional nomenclature of process control and performance simulation $\rho(\xi, t)$ represents local WIP density and the flux $\rho(\xi, t) v(\xi, t)$ is the local throughput at stage $\xi$ at time $t$, respectively. As a starting point for the state equation it has to respect the fundamental law of factory physics, Little's law [26]. For a single factory Little's law may be written as

$$
N=\tau \lambda,
$$

where $N$ is the time-averaged load of the factory (WIP), $\tau$ is the mean cycle or throughput time over all outputs (TPT) and $\lambda$ is the start rate. Little's law is fundamentally a deterministic law and results from mass conservation. However, by amending it with a description of the stochastic processes in a factory we can generate a state equation characterizing the factory. In its simplest case the stochastic process is represented by its means. For instance, modeling a factory as a single linear queue with Markov arrival and processing rates (an $M / M / 1$ queue) the mean cycle time $\tau$ can be determined as a function of the start rate $\lambda$ and processing rate $\mu[13]$ to be

$$
\tau=\frac{1}{\mu-\lambda}
$$

Therefore, the relationship between WIP and cycle time becomes

$$
\tau=\frac{1}{\mu}(1+N) .
$$


Equation (1.14) shows that $\mu$ becomes the maximal or critical start rate. With $v=1 / \tau$ we have the desired state equation. Equation (1.15) shows that WIP is a linear function of the cycle time with a slope $\mu$. The linear relationship between TPT and WIP is intuitively obvious: a part entering the queue will have to wait until the queue is served ( $N / \mu$ time units) plus its own processing time, $1 / \mu$ time units.

A linear relationship as in Eq. (1.15) is true for a queueing network that has product form $[29,6]$, i.e. the whole network can be replaced by an effective queue. However there are several key factors that will lead to a queueing network that cannot be approximated by a product network with constant production rates. Specifically, semiconductor production lines are re-entrant with highly complex topologies. As a result they show moving bottlenecks and other nonlinear behavior. In addition, there is anecdotal evidence from real re-entrant factories [17] that show a stronger than linear increase in the average throughput time $\tau$ as the loading of the factory is increased. In addition, Lu et al. [27] show that through dispatch and scheduling rules one can reduce the variance of cycle times inside semiconductor manufacturing plants while Dai and Weiss [11] show that some scheduling rules lead to unstable networks, i.e. WIP grows to infinity. Furthermore, operator fatigue will lead to increased error rates as the load in the factory is increased. As a result, the network cannot be approximated by a product network with constant production rates any more but rather through queues whose production rate depends on the length of the queue. Unfortunately, the true state equation for a re-entrant factory is not known, as large factories are rarely (if ever) run in equilibrium and controlled experiments are obviously impossible. It is worth noting here that discrete event simulators are not a good substitute for real experiments specifically if human interaction is part of the cause for the increase in variance.

In order to develop the type of results that can be achieved with a continuum-model approach based on a conservation-law model and to show the feasibility of fast simulations for large production and supply networks we choose a specific model for a re-entrant factory. We therefore assume a state equation analogous to the Lighthill-Whitham traffic model of the form

$$
v(\rho)=v_{0}\left(1-\frac{\bar{\rho}(t)}{L}\right),
$$

with

$$
\bar{\rho}(t)=\int_{0}^{1} \rho(\xi, t) d \xi .
$$

Here $v_{0}$ is the speed for the empty factory and $L$ is the maximal load (capacity of the factory). All the following analysis can easily be adapted to fit any other state equation that has been validated through real data or justified through heuristic arguments. As a result of Eq. (1.16) the throughput time, instead of showing a singularity with respect to the start rate, has a singularity with respect to WIP, i.e.

$$
\tau=\frac{\tau_{0}}{L-\bar{\rho}}
$$

where $L$ is called the critical load. Clearly the TPT increases without bound as $\bar{\rho} \rightarrow L$. This implies that the factory can only run in equilibrium for WIP less than $L$. It is instructive to 


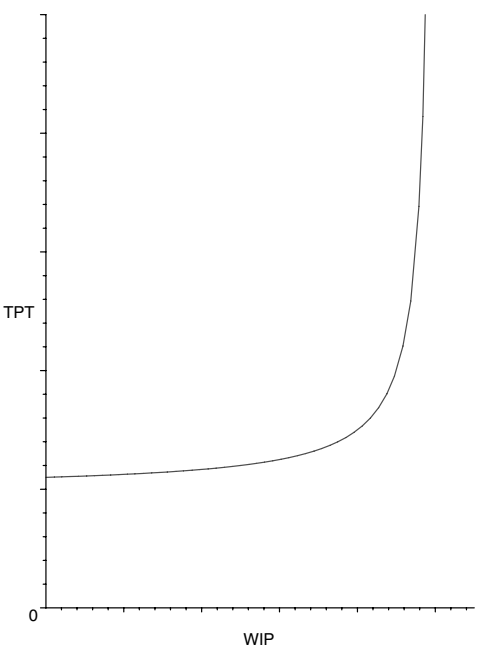

(a)

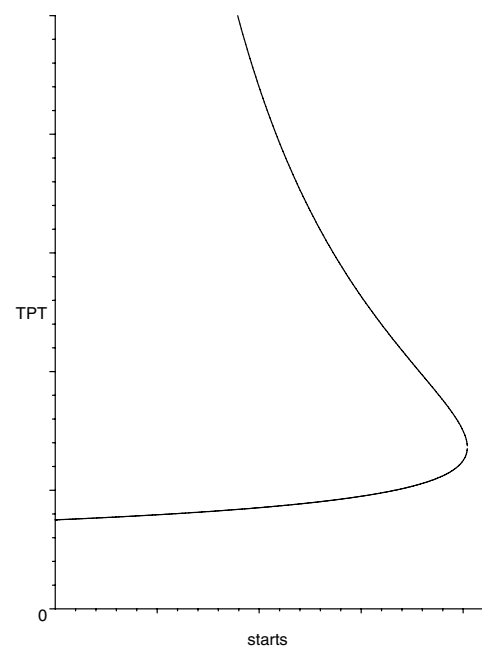

(b)

Figure 1.6: (a) Schematic of TPT as a function of WIP, Eq. (1.17), (b) TPT as a function of start rate.

input Eq. (1.17) into Little's law: Fig. 1.6 shows TPT as a function of WIP (Eq. (1.17)) and TPT as a function of start rate. Notice that there is still a critical start rate $\lambda_{\mathrm{c}}$ : For $\lambda<\lambda_{\mathrm{c}}$ there exist two equilibria, for $\lambda>\lambda_{\mathrm{c}}$ there are none, a case of a saddle-node bifurcation.

There is a crucial difference between the state equation for our factory models, Eq. (1.17), and the Lighthill-Whitham model for traffic flow. Our factory models represent re-entrant factories. Therefore, as a first-order model, the velocity is uniform in the whole factory and a local density fluctuation influences the velocity everywhere. In contrast, traffic velocity depends on the local density only. A more accurate model could use a state equation of the form

$$
v(\xi, t)=v_{0}\left(1-\frac{\int_{0}^{1} w(\xi, s) \rho(s, t) d s}{L}\right) .
$$

Here appropriate weight functions $w(\xi, s)$ describe the influence of WIP at stage $s$ on the velocity of WIP at stage $\xi$ and can be used to model in more detail the complicated topologies of re-entrant product flow.

To conclude, we re-state the full model, with $g(\xi)$ an initial density distribution, and taking into account that $v$ as in Eq. (1.17) does not depend on $\xi$ :

$$
\begin{aligned}
& \rho_{t}+v(\rho) \rho_{\xi}=0, \\
& v(\rho)=v_{0}\left(1-\frac{\bar{\rho}(t)}{L}\right), \\
& \rho(\xi, 0)=g(\xi), \\
& \rho(0, t) v(t)=\lambda(t) .
\end{aligned}
$$


Notice that the start rate $\lambda(t)$ into the factory enters as the boundary condition for the local throughput at $\xi=0$.

This model shares many features with thermodynamic transport equations. In particular, modeling the relationship between density and velocity through a state equation is typically called an adiabatic approximation: factory flow is modeled as if it were always in equilibrium, following adiabatically the state equation (1.17). As a result, we can expect that fast transients will be modeled poorly but that slow transients and averages will be modeled very well.

A more elaborate model including a dynamic equation for the evolution of the velocity is presented in [2]. Here we derive the Boltzmann or Euler equations for the transport along a factory and generate an additional conservation law for the time evolution of the velocity. Modeling a factory or part of a factory as a single queue (linear flow, not re-entrant), we derive

$$
\begin{aligned}
& \rho_{t}+(v(\rho) \rho)_{\xi}=0, \\
& v_{t}+v v_{\xi}=0,
\end{aligned}
$$

with boundary conditions

$$
\begin{aligned}
& v(0, t)=\frac{v_{0}}{1+\bar{\rho}(t)}, \\
& \bar{\rho}(t)=\int_{0}^{1} \rho(\xi, t) d \xi, \\
& v(0, t) \rho(0, t)=\lambda(t),
\end{aligned}
$$

where $\lambda(t)$ is the prescribed influx into the factory and $1 / v_{0}$ is the production rate of the queue.

\subsubsection{Simulation and Control}

Modeling production flow through conservation laws of hydrodynamic type opens up the vast literature of scientific computing for these equations (e.g. [23, 24]). As a result, fast and accurate simulations of Eq. (1.18) or Eq. (1.19) are straightforward. In addition, theoretical analysis of Eq. (1.18) can be done. For instance, linear stability analysis of the two equilibria of Fig. 1.6 leads to a linear delay equation with the high-speed-low-WIP equilibrium being stable and the low-speed-high-WIP equilibrium being unstable.

The method of characteristics can be used to solve the following control problem: change the production flow of the factory from one steady state, corresponding to an outflux that meets a specific constant demand, to another one. We assume that the demand $d(t)$ changes like

$$
d(t)= \begin{cases}d_{1} & t<0 \\ d_{2} & t>0\end{cases}
$$

Due to the production lag, any change in the start of the factory will leave the factory at some later time. Hence there will be a mismatch of the desired output $d_{2}$ and the actually produced output at least until the change in influx has moved through the factory. We call that mismatch backlog. The control problem is to design an influx $\lambda(t)$ that would move the system from the equilibrium $\rho_{1}$ corresponding to a production rate of $d_{1}$ to a new equilibrium $\rho_{2}$ corresponding to a production rate $d_{2}$, generating an output such that the backlog becomes zero in the shortest possible time. We show in [1] how to arrive at the following heuristic: 
If the jump in demand is small (leading to a small enough backlog) then the optimal strategy will be to get the factory to its maximal density instantly and keep it there for the right amount of time, such that when this extra product leaves the factory, the backlog is zero.

It is an open problem to design an optimal control strategy for all jumps in demand. Simulations and control experiments can be viewed on the webpage http://math. duke. edu/ daniel/supply_chain.html.

\section{Acknowledgment}

This paper reviewed collaborative work with Esma Gel, Karl Kempf, Dan Marthaler, Junko Murakami, Bart Rem and Christian Ringhofer. Support through grants from NSF (DMS0204543) and from Intel Corporation is gratefully acknowledged.

\section{Bibliography}

[1] D. Armbruster, D. Marthaler, and C. Ringhofer, A continuum model for a re-entrant factory, preprint, Arizona State University (2002).

[2] D. Armbruster, D. Marthaler, C. Ringhofer, Kinetic and fluid model hierarchies for supply chains, to appear in SIAM MMS (2003).

[3] D. Armbruster, E.S. Gel, Bucket Brigades Revisited: Are they always effective? preprint, Arizona State University (2003).

[4] D. Armbruster, E.S. Gel, and J. Murakami, Bucket brigades and learning presented at INFORMS 2002, San Jose, paper in preparation (2003).

[5] J.J. Bartholdi and D.D. Eisenstein, A production line that balances itself, Operations Research 44(1) (1996), 21-34.

[6] F. Baskett, K.M. Chandy, R.R. Muntz, and F.G. Palacios, Open, closed and mixed networks of queues with different classes of customers, Journal of the Association of Computing Machinery 22 (1975), 248-260.

[7] D.P. Bischak, Performance of a manufacturing module with moving workers, IIE Transactions 28 (1996), 723-733.

[8] L.A. Bunimovich, Method of stabilization of a target regime in manufacturing and logistics, Chapter 2, this book.

[9] C.J. Chase, J. Serrano, and P.J. Ramadge, Periodicity and chaos from switched flow systems: contrasting examples of discretely controlled continuous systems, IEEE Transactions on Automatic Control 38(1) (1993), 70-83.

[10] H. Chen, J.M. Harrison, A.Mandelbaum, A. Van Ackere, and L.M. Wein, Empirical evaluation of a queueing network model for semiconductor wafer fabrication, Operations Research 36 (1988), 202-215.

[11] J.G. Dai and G. Weiss, Stability and isntability of fluid models for certain re-entrant lines, Mathematics of Operations Research 21 (1996), 115-134.

[12] See e.g. Factory Explorer, WWK products (1996). 
[13] D. Gross and C.M. Harris, Fundamentals of Queueing Theory, Wiley, New York, 1985.

[14] D. Helbing, Modeling and optimization of supply networks: Lessons from traffic dynamics, Chapter 6, this book.

[15] C. Horn and P.J. Ramadge, A topological analysis of a family of dynamical systems with non-standard chaotic and periodic behavior, International Journal of Control 67(6) 1997, 979-996.

[16] F.M. Bevis and D.R. Towill, Continued learning and the work study observer, Work Study and Management Services 18 (1974), 420-427.

[17] K. Kempf, Intel, personal communications (2001).

[18] I. Katzorke and A. Pikovsky, Chaos and complexity in simple models of production dynamics, Discrete Dynamics in Nature and Society 5 (2000), 179-187.

[19] P.R. Kumar, Re-entrant lines, Queueing Systems 13 (1993), 87-110.

[20] E. Lefeber, Nonlinear models for control of manufacturing systems, Chapter 5, this book.

[21] R.I. Leine, D.H. Van Campen, and B.L. Van der Vrande, Bifurcations in nonlinear discontinuous systems, Nonlinear Dynamics 23 (2000), 105-164.

[22] P. Lendermann, B.P. Gan, and L.F. McGinnis, Distributed simulation with incorporated APS procedures for high-fidelity supply chain optimization, in: Proceedings of the 2001 Winter Simulation Conference, Washington DC (2001), pp. 1138-1145.

[23] R.J. LeVeque, Finite Difference Methods for Differential Equations. Draft version for use in AMath 585-6, University of Washington (1998).

[24] R.J. LeVeque, Numerical Methods for Conservation Laws, Birkhäuser-Verlag, Basel, 1992.

[25] M.J. Lighthill and G.B. Whitham, On kinematic waves II. A theory of traffic flow on long crowded roads, Proceedings of the Royal Society, Series A, 229 (1955), 317-345.

[26] J.D.C. Little, A proof for the queuing formula $L=\lambda W$, Operations Research 9 (1961), 383-387.

[27] S.C.H. Lu, D. Ramaswamy, and P.R. Kumar, Efficient scheduling policies to reduce mean and variance of cycle-time in semiconductor manufacturing plants, IEEE Transactions on Semiconductor Manufacturing 7 (1994), 374-385.

[28] G.F. Newell, Approximation methods for queues with application to the fixed-cycle traffic light, SIAM Review 7(2) (1965), 223-240.

[29] R. Nelson, Probability, Stochastic Processes, and Queueing Theory, Springer Verlag, New York, 1995.

[30] G.F. Newell, Scheduling, location, transportation and continuum mechanics; some simple approximations to optimization problems, SIAM Journal of Applied Mathematics 25(3) (1973), 346-360.

[31] Scalable Enterprise Initiative, NSF 2000.

[32] E. Ott, C. Grebogi, and J.A. Yorke, Controlling Chaos, Phys. Rev. Lett. 64 (1990), 3215.

[33] K. Peters, J. Worbs, U. Parlitz, and H.-P.Wiendahl, Manufacturing systems with restricted buffer sizes, Chapter 3, this book.

[34] A. Pikovsky, M. Rosenblum, and J. Kurths, Synchronization, Cambridge University Press, 2001. 
[35] B. Rem and D. Armbruster, Control and synchronization in switched arrival systems, Chaos 13(1) (2003), 128-137.

[36] B. Scholz-Reiter, M. Freitag, A. Schmieder, A, Pikovsky, and I. Katzorke, Modelling and analysis of a re-entrant manufacturing system, Chapter 4 , this book.

[37] S.N. Simic, K.H. Johansson, S. Sastry, and J. Lygeros, Towards a geometric theory of hybrid systems, in: Hybrid Systems: Computation and Control, edited by N. Lynch and B.H. Krogh, LNCS 1790, pp. 421-436, Springer, 2000.

[38] T. Ushio, H. Ueda, and K. Hirai, Controlling chaos in a switched arrival system, Systems \& Control Letters 26 (1995), 335-339.

[39] T. Ushio, H. Ueda, and K. Hirai, Control of Chaos in Switched Arrival Systems with N Buffers, Electronics and Communications in Japan, Part 3, 83(8) (2000), 81-86. 